\title{
Sensitivities of surface wave velocities to the medium parameters in a radially anisotropic spherical Earth and inversion strategies
}

\author{
Sankar N. Bhattacharya
}

Lithosphere Study Centre, New Delhi, India

\author{
Article history \\ Received June 14, 2015; accepted September 23, 2015. \\ Subject classification: \\ Crust-mantle structure, Radial anisotropy, Surface waves.
}

\begin{abstract}
Sensitivity kernels or partial derivatives of phase velocity (c) and group velocity $(\mathrm{U})$ with respect to medium parameters are useful to interpret a given set of observed surface wave velocity data. In addition to phase velocities, group velocities are also being observed to find the radial anisotropy of the crust and mantle. However, sensitivities of group velocity for a radially anisotropic Earth have rarely been studied. Here we show sensitivities of group velocity along with those of phase velocity to the medium parameters $\mathrm{V}_{\mathrm{SV}}, \mathrm{V}_{\mathrm{SH}}, \mathrm{V}_{\mathrm{PV}}, \mathrm{V}_{\mathrm{PH}}$, $\eta$ and density in a radially anisotropic spherical Earth. The peak sensitivities for $\mathrm{U}$ are generally twice of those for $\mathrm{c}$; thus $\mathrm{U}$ is more efficient than c to explore anisotropic nature of the medium. Love waves mainly depends on $\mathrm{V}_{\mathrm{SH}}$ while Rayleigh waves is nearly independent of $\mathrm{V}_{\mathrm{SH}}$. The sensitivities show that there are trade-offs among these parameters during inversion and there is a need to reduce the number of parameters to be evaluated independently. It is suggested to use a nonlinear inversion jointly for Rayleigh and Love waves; in such a nonlinear inversion best solutions are obtained among the model parameters within prescribed limits for each parameter. We first choose $\mathrm{V}_{\mathrm{SH}}, \mathrm{V}_{\mathrm{SV}}$ and $\mathrm{V}_{\mathrm{PH}}$ within their corresponding limits; $\mathrm{V}_{\mathrm{PV}}$ and $\eta$ can be evaluated from empirical relations among the parameters. The density has small effect on surface wave velocities and it can be considered from other studies or from empirical relation of density to average $P$ wave velocity.
\end{abstract}

\section{Introduction}

A transversely isotropic elastic medium with vertical axis of symmetry or radially anisotropic medium is described by the medium parameters: density $\rho$, wave velocity of horizontally and vertically travelling P-waves $V_{P H}$ and $V_{P V}$, vertically and horizontally polarised $S$ waves $V_{S V}$ and $V_{S H}$ and the parameter $\eta$ as described by Takeuchi and Saito [1972]. These parameters are linked with 5 elastic moduli as $A=\rho V_{P H}^{2}, C=\rho V_{P V}^{2}, L=\rho V_{S V}^{2}$, $N=\rho V_{S H}^{2}$ and $F=\eta \rho\left(V_{P H}^{2}-2 V_{S V}^{2}\right)$. A seismic anisotropy is created by fabric induced from deformation in the crust and mantle; this causes dependence of local wave propagation on the direction and polarization of wave field [Chen et al. 2009]. A layer with $V_{S H}>V_{S V}$ associated with horizontal flow such as underneath plates; a vertical flow creates $V_{S H}<V_{S V}$ as underneath spreading centres or subduction zones. Thus knowledge of seismic anisotropy allows us to understand the Earth's dynamic evaluation.

Radial anisotropy in the uppermost mantle was considered to explain the fundamental modes of both Rayleigh and Love wave phase velocities, particularly, when isotropic structure failed to do so [Anderson 1961, McEvilly 1964]. Several such results led to incorporation of radial anisotropy in the top $220 \mathrm{~km}$ of the mantle in Preliminary Reference Earth Model (PREM; Dziewonski and Anderson [1981]). Since then surface wave studies have confirmed such anisotropy in many regions [e.g. Villasenor et al. 2001, Bhattacharya et al. 2013] and might be deeper in some cases [Visser et al. 2008]. With the availability of broadband data globally, 3-D global maps of radial anisotropy of uppermost mantle have been prepared [Panning and Romanowicz 2006, Kustowski et al. 2008].

Radial anisotropy in the crust has also been observed in Himalaya and Tibet [Shapiro et al. 2004, Xie et al. 2013] and SE Europe [Raykova and Nikolova 2003]. Radial anisotropy in the crust has been noted in the extensional provinces such as, western United States [Moschetti et al. 2010] and off-coast of western India [Prajapati et al. 2011].

Sensitivity study of surface wave velocity to medium parameters is important to interpret a set of observed surface wave velocity data. Changes in period in a given mode of spheroidal and toroidal oscillations to medium parameters of a radially anisotropic Earth were shown by Anderson [1989] and Montagner [2007]. Sensitivities of phase velocity at different periods to such medium parameters were shown in a few studies [e.g. Takeuchi and Saito 1972]. Muyzert and Snieder [2000] compared 
phase velocity sensitivities to Love parameters $\left[\mathrm{m}_{\mathrm{L}}\right]=$ $[A, C, L, N, \mathrm{~F}, \rho]$ and those to Anderson parameters $\left[\mathrm{m}_{\mathrm{A}}\right]$ $=\left[V_{S V}, V_{S H}, V_{P V}, V_{P H}, \eta, \rho\right]$.

Thus there are a few studies on sensitivities of phase velocities but rarely any study on those of group velocities. In recent years group velocities are also being used to find the radial anisotropy of the crust and mantle [e.g. Shapiro and Ritzwoller 2002, Shapiro et al. 2004, Chen et al. 2009]. To fill up the gap we show here the sensitivities of group velocity along with those of phase velocity of fundamental mode to medium parameters $\left[\mathrm{m}_{\mathrm{A}}\right]$ in a spherical Earth. We also suggest a few inversion strategies based on these sensitivities.

\section{Computations of sensitivities}

For Rayleigh wave, Bhattacharya and Arora [1997] obtained dispersion equation in a radially anisotropic layered spherical Earth considering each layer to be heterogeneous as

$$
[\mathrm{A}, \mathrm{C}, \mathrm{L}, \mathrm{N}, \mathrm{F}, \rho]=\left[\mathrm{A}_{0}, \mathrm{C}_{0}, \mathrm{~L}_{0}, \mathrm{~N}_{0}, \mathrm{~F}_{0}, \rho_{0}(r / a)^{-2}\right](r / a)^{-2}
$$

where $\mathrm{A}_{0}, \mathrm{C}_{0}, \mathrm{~L}_{0}, \mathrm{~N}_{0}, \mathrm{~F}_{0}$ and $\rho_{0}$ are constants, $a$ is the radius of the Earth and $r$ is the radial distance from the centre of the Earth. Considering the layer thickness to be small compared to the wavelength, the layer behaves nearly like a homogeneous layer [Bhattacharya 1976]. Advantage of such small heterogeneity is to get solutions of the equations of motions in terms of exponential functions making the dispersion equation as simple as that for a flat layered Earth. We call 'exact flattening transformation' since we use the exact solutions of equation of motion in terms of exponential functions as in a flat Earth. The dispersion equation is obtained from that of a flat layered Earth by changing displacementstress matrix [Bhattacharya and Arora 1997].

For Love waves, equation of motion is simple [Takeuchi and Saito 1972] and its solution is obtained in terms of exponential functions with same heterogeneity as (1) in a layer. The dispersion equation of Love waves through exact flattening transformation can be obtained by changing displacement-stress matrix of a flat layered Earth as done for an isotropic case [Bhattacharya 2009]. The displacement-stress matrix of radially anisotropic spherical Earth with flattening transformation will be same as Bhattacharya ([2009]; Eq. (7) with $p=-2$ ) by replacing $\mu_{0}$ by $L_{0}$ and considering $\nu_{\beta}^{2}$ as

$$
v_{\beta}^{2}=-\frac{\omega^{2} \rho_{0}}{L_{0}}+\frac{N_{0}}{L_{0}}\left(k^{2}-\frac{9}{4 a^{2}}\right)+\frac{1}{4 a^{2}}
$$

Let us consider the dispersion equation as $f(k, \omega)=$ 0 , where $k$ is the wave number and $\omega$ is the angular fre- quency. For a given value of period $(T=2 \pi / \omega)$, phase velocity $(c=\omega / k)$ is obtained as a root of the dispersion equation through bisection method. The group velocity $(U)$ is obtained as [Stein and Wysession 2003]

$$
U=\frac{d \omega}{d k}=-\frac{\left(\partial f^{\prime} / \partial k\right)_{\omega}}{\left(\partial f^{\prime} / \partial \omega\right)_{k}}
$$

where the above partial derivatives in the numerator and denominator at a root are obtained from analytical expressions of $f$.

To compute the sensitivities or partial derivatives of $c$ and $U$ with respect to each model parameter of a given crust-mantle structure, we consider PREM, which represents an average Earth model. For a particular region such sensitivities can be drawn in a similar manner. PREM has $3 \mathrm{~km}$ oceanic layer and crust extends down to $24.5 \mathrm{~km}$. Between depths 24.5 and $220 \mathrm{~km}$, the model is radially anisotropic; but we have made this zone isotropic with effective isotropic velocities as suggested by Dziewonski and Anderson [1981]. Thus we consider a radially anisotropic PREM with simplification that $V_{S V}=V_{S H}, V_{P V}=V_{P H}$, and $\eta=1$ (Figure 1). Although such simplification makes the model to be isotropic, however the sensitivities each parameter of $\left[\mathrm{m}_{\mathrm{A}}\right]$ will show how $c$ and $U$ changes as each parameter changes from isotropic to radially anisotropic as done for $c$ in Muyzert and Snieder [2000]; however, $\rho$ changes show sensitivities for an isotropic PREM model. We change each element of $\left[\mathrm{m}_{\mathrm{A}}\right]$ in a layer of $1 \mathrm{~km}$ centred at a given depth to get the following dimensionless

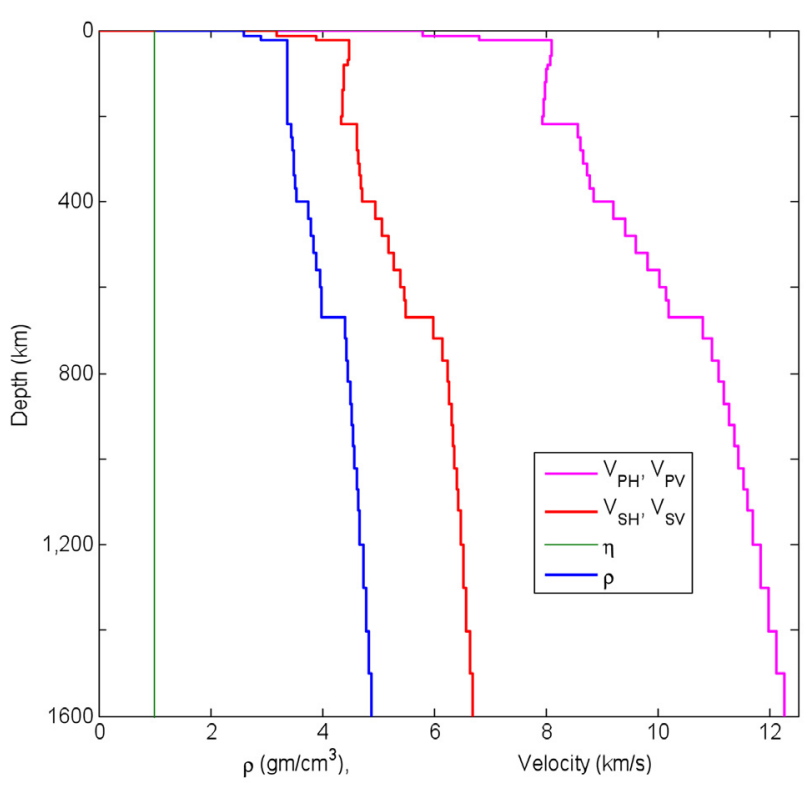

Figure 1. PREM with effective isotropic velocities at $100 \mathrm{~s}$ period [Dziewonski and Anderson 1981]. We change each elements of $\left[\mathrm{m}_{\mathrm{A}}\right]=\left[V_{S V}, V_{S H}, V_{P V}, V_{P H}, \eta, \rho\right]$ to get the corresponding partial derivatives in Figures 2 and 3. 
partial derivatives for a given period $T$ by numerical differentiation

$$
\left[\frac{\partial v}{\partial V_{S V}}, \frac{\partial v}{\partial V_{S H}}, \frac{\partial v}{\partial V_{P V}}, \frac{\partial v}{\partial V_{P H}}, \frac{1}{c_{T}} \frac{\partial v}{\partial \eta}, \frac{\rho_{m}}{c_{T}} \frac{\partial v}{\partial \rho}\right]
$$

where $v=$ either $c$ or $U ; \rho_{m}$ is the density in the mantle just below Moho and $c_{T}$ is the phase velocity at the pe$\operatorname{riod} T$. The Love wave is independent of $V_{P V}, V_{P H}$ and $\eta$. We use $\rho_{m}=3.38 \mathrm{gm} / \mathrm{cm}^{3}$. We obtain partial derivatives of fundamental mode $c$ and $U$ at periods 20, 50, 100 and $200 \mathrm{~s}$ (Figures 2 and 3)

\section{Discussions}

The sensitivities or partial derivatives shown in Figures 2 and 3 allow us to find the depth to which we can explore and find a strategy to improve the exploration with a given set of observed surface wave velocity data. Both for Rayleigh and Love waves, peak partial derivatives of $U$ are normally twice of those of $c$; this makes $U$ more useful than $c$. However, measurement of phase velocity is more accurate than that of group velocity in a few cases [Suresh et al. 2015]. The partial derivatives of $U$ to velocities have peaks in the positive and negative sides with a deepest zero crossing at a depth about $\lambda / 2$ for Rayleigh waves and about $\lambda / 3$ for Love waves, where $\lambda\left(=T * c_{T}\right)$ is the wavelength; below this depth the partial derivatives are small. The deepest zero crossing depths of sensitivities for all the parameters are close to each other. At zero-crossing depth of a period, the medium parameters are explored by other periods for which sensitivities are non-zero at that depth. Thus with a given set of group velocity data, we can approximately explore down to the deepest depth of zero crossing of the highest period available in the set. For Rayleigh waves, depth of peak sensitivity increases with period; but for Love waves it slightly increases with period but mostly remains in the crust and close to Moho. In isotropic case, similar characteristics of sensitivities to $V_{S}$ were noted [Levedev et al. 2013]. In an isotropic Earth, Dahlen and Tromp [1998] noted that phase velocities feel perturbations of $V_{S}$ down to $\sim \lambda / 2$ for Rayleigh waves and $\sim \lambda / 4$ for Love waves. The large values of sensitivities of Rayleigh and Love waves to the model parameters of crust and close to Moho necessitates us to evaluate parameters of this zone carefully while exploring deeper part [Ferreira et al. 2010, Panning et al. 2010].

As is well known, Love waves do not depend on $V_{P V}, V_{P H}$ and $\eta$. Sensitivities of Rayleigh wave velocity to $V_{P V}$ and $V_{P H}$ are substantial. These two sensitivities are generally of opposite signs at given depth; thus increase of one and decrease of other affect surface wave velocity as significantly as by change of $V_{S V}$ [Anderson 1989]. For an isotropic case, opposite signs of sensitivities cancel approximately and sensitivity of $V_{P}$ is only about 10 per cent of that to $V_{S}$ [Suresh et al. 2015].

For Love waves, sensitivity to $V_{S H}$ is large but negligible to $V_{S V}$. Thus the velocities of Love waves computed in an isotropic medium with $V_{S}$ is nearly same as those computed in a radially anisotropic medium with $V_{S H}=V_{S}$ and $V_{S V}$ may have any value and may be obtained from Rayleigh wave inversion. On the other hand, Rayleigh wave is nearly indifferent of $V_{S H}$. Hence the velocities of Rayleigh waves computed in an isotropic medium with $V_{S}$ and $V_{P}$ will be nearly same as those computed in a radially anisotropic medium with $V_{S V}=V_{S}$ and $V_{S H}$ may be have any value and may be obtained from Love wave inversion; here we assume $V_{P V}=V_{P H}=V_{P}$ and $\eta=1$. This criteria have been used by a few authors while inverting radial anisotropy [Raykova and Nikolova 2003, Bjarnason and Schmeling 2009, Prajapati et al. 2011]. Although such computation using computational codes of a isotropic medium is nearly correct for Love waves, but for Rayleigh waves the assumption of an anisotropic medium with $V_{S V} \neq$ $V_{S H}, V_{P V}=V_{P H}$ and $\eta=1$ is not strictly correct [Babuska and Cara 1991, Xie et al. 2013]. In real mineral assemblages, $V_{S}$ anisotropy would be accompanied by $V_{P}$ anisotropy with $\eta \neq 1$. Further, assumption of $V_{P V}=$ $V_{P H}$ may over estimate of $S$-wave anisotropy because part of changes in phase / group velocities by changing $V_{S V}$ may be obtained by changing $V_{P V}$ and $V_{P H}$.

The sensitivity to the fifth parameter $\eta$ is also significant. Figure 2 shows $(\partial v / \partial \eta) / c_{T}$; the factor $1 / c_{T}$ has been considered to make the sensitivity dimensionless. Density $\rho$ of the crust mainly influences Love wave velocities $(c$ or $U$ ) at all periods. Sensitivity of Rayleigh wave velocity to $\rho$ is small but significant; depth of peak sensitivity increases with period. However, it is felt that we should restrict the inversion to first five parameters and density can be obtained from gravity studies or from an empirical formula using average P-wave velocity.

As stated above, observed Love wave velocities can be easily inverted to give $V_{S H}$. For Rayleigh waves, the sensitivities of velocities $c$ and $U$ to $V_{S V}, V_{P V}, V_{P H}$ and $\eta$ show trade-offs; i.e. increase or decrease of one will have nearly same effect on velocities by increase or decrease of the others. So even independent evaluation of all $V_{S V}, V_{P V}, V_{P H}$ and $\eta$ from Rayleigh waves may not give correct result. It is suggested that we can explore two parameters: $V_{S V}$, and either $V_{P V}$ or $V_{P H}$. The remaining two may be obtained from empirical relations of real mineral assemblages such as [Xie et al. 2013]

$$
\varepsilon \approx 0.5 \gamma, \quad \eta \approx 1.0-4.2 \gamma
$$



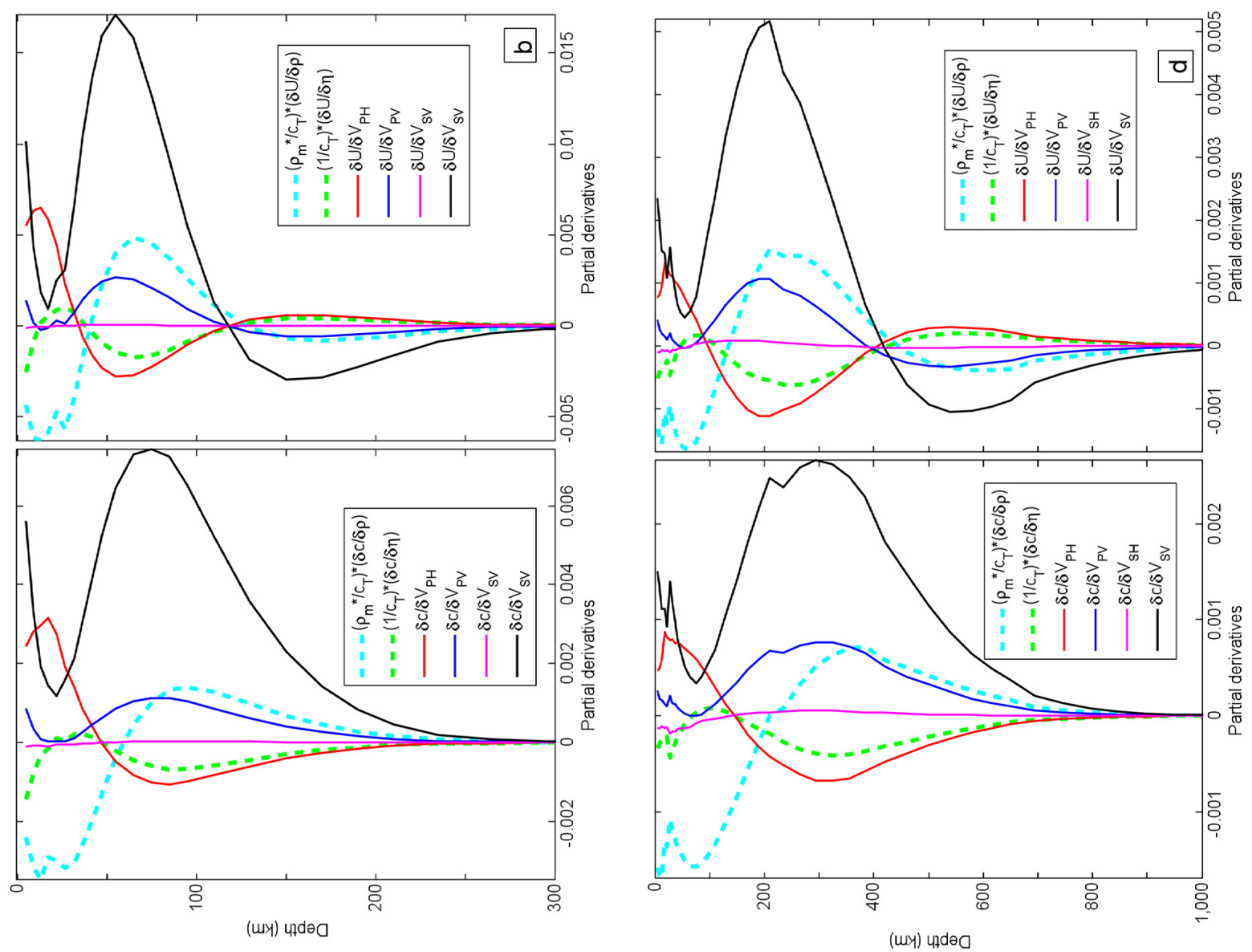

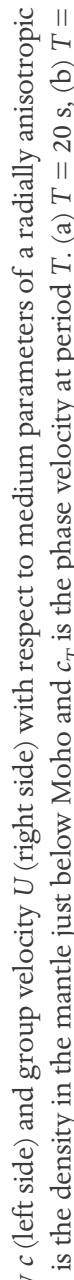
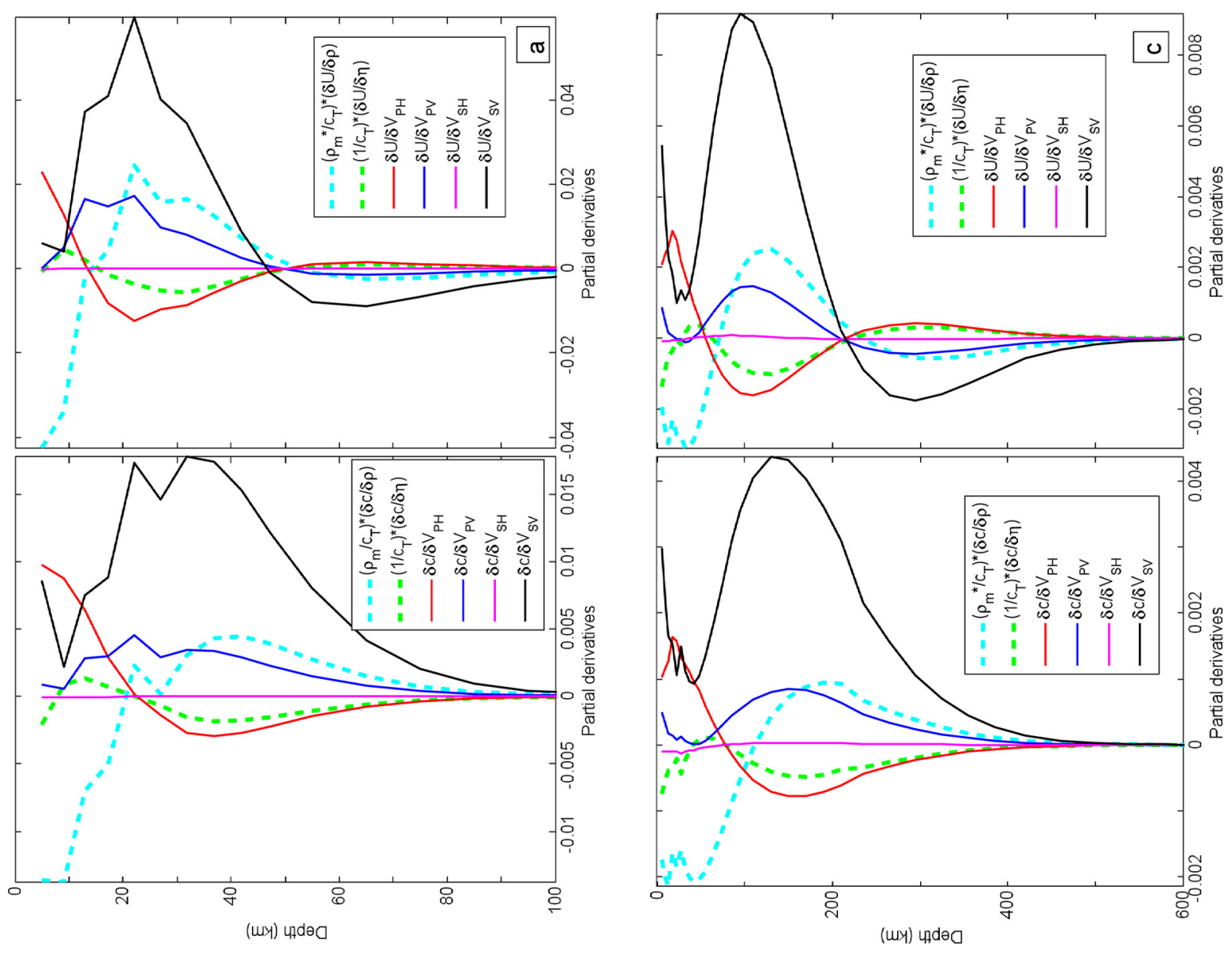

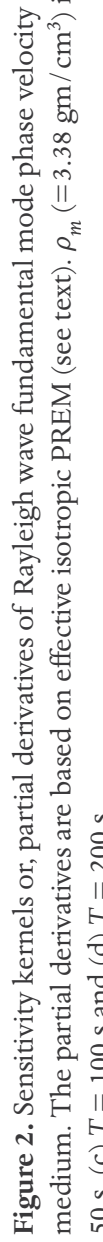



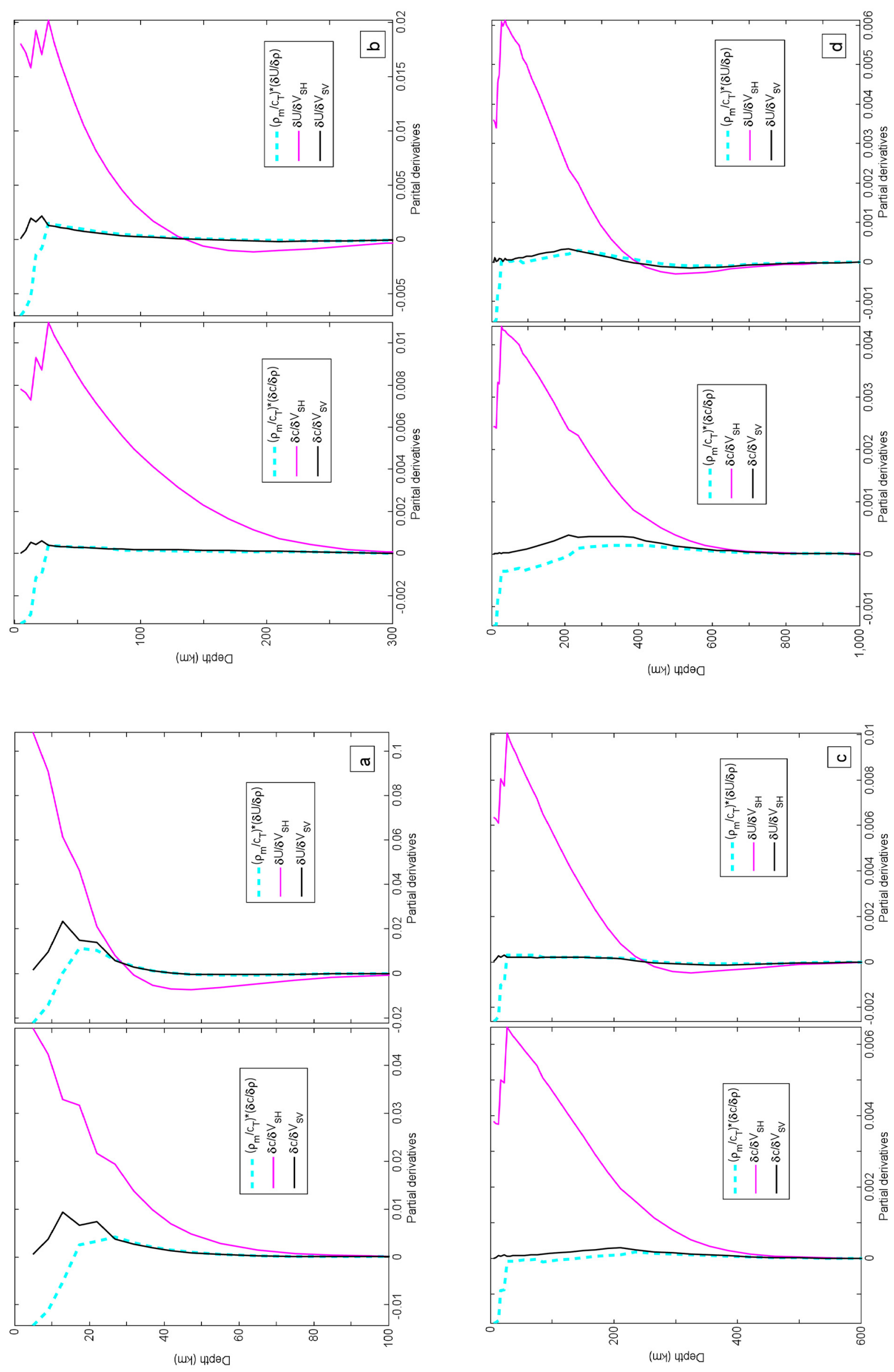

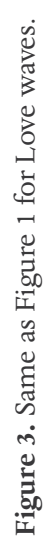


where

$\gamma=\left(V_{S H}-V_{S V}\right) / V_{S}, \varepsilon=\left(V_{P H}-V_{P V}\right) / V_{P}$

and effective Voigt average speed [Babuska and Cara 1991]

$V_{S}=\sqrt{ }\left[\left(2 V_{S V}^{2}+V_{S H}^{2}\right) / 3\right]$ and $V_{P}=\sqrt{ }\left[\left(V_{P V}^{2}+4 V_{P H}^{2}\right) / 5\right]$.

We write $(3 \mathrm{~b})$ as

$$
V_{P V}=V_{P H}-\varepsilon V_{P}
$$

We first consider values of $V_{S H}, V_{S V}$ and $V_{P H}$ The parameter $\eta$ can be obtained from (2b) after obtaining $\gamma$ from (3a). We preferred first to consider $V_{P H}$ rather than $V_{P V}$ because as per Equation (4b), we may approximate $V_{P} \approx V_{P H}$. Thus from (5) we get $V_{P V} \approx(1-\varepsilon) V_{P H}$ as a first approximation. Substituting this expression of $V_{P V}$ in (4b) we get second approximation of $V_{P}$ as

$$
V_{P} \approx(1-\varepsilon / 5) V_{P H}
$$

Substituting in (5), we get second approximation of $V_{P V}$ as

$$
V_{P V} \approx\left(1-\varepsilon+\varepsilon^{2} / 5\right) V_{P H}
$$

To evaluate radial anisotropy, Love and Rayleigh waves need to be inverted jointly with nonlinear inversion where the best solution are obtained among the model parameters within prescribed upper and lower limits for each parameter [Suresh et al. 2008, Prajapati et al. 2011]. For these model parameters, we first choose $V_{S H}, V_{S V}$ and $V_{P H}$ within their corresponding limits and the remaining parameters $V_{P V}, \eta$ and $\rho$ can be obtained as discussed above.

\section{Conclusions}

(i) Love wave velocities in a radially anisotropic medium are close to those in an isotropic medium with $V_{S}=V_{S H}$. The $V_{S}$, obtained from inversion of only Love wave velocities through isotropic code, is close to $V_{S H}$, if the medium is radially anisotropic.

(ii) However, Rayleigh wave velocities in a radially anisotropic medium can only be approximated by an isotropic code with $V_{S}=V_{S V}$ under the assumption that $V_{P V}=V_{P H}=V_{P}$ and $\eta=1$; this assumption is not strictly correct if $V_{S V} \neq V_{S H}$. Further assumption of $V_{P V}=V_{P H}$ may cause over estimation of $S$-wave anisotropy.

(iii) Sensitivity being greater, group velocity is more efficient than phase velocity to explore anisotropic nature of the medium. The depth limit of inversion is ascertained by the deepest depth of zero crossing of the sensitivities at the highest period available in the data set. This depth is about half the wavelength for Rayleigh waves and about one-third of wavelength for Love waves

(iv) Through Rayleigh waves, both $V_{P V}$ and $V_{P H}$ in radially anisotropic medium are better explored than exploring $V_{P}$ in an isotropic medium.

(v) $V_{S H}$ is evaluated from Love waves and this evaluation is nearly independent of Rayleigh waves. It is evaluated well for the lithosphere below which the sensitivity of Love wave velocity to $V_{S H}$ rapidly decreases.

(vi) To evaluate radial anisotropy, we need a joint nonlinear inversion of observed velocities of Rayleigh and Love waves. In such nonlinear inversion the best solutions are chosen among the model parameters, each within given upper and lower limits. We first choose $V_{S H}, V_{S V}$ and $V_{P H}$ within their corresponding limits and $V_{P V}$ and $\eta$ are obtained from empirical relations. The density $(\rho)$ has only small effect on surface waves and it can be considered from other studies or can be obtained using the empirical relation of density with average P-wave velocity at that depth.

\section{References}

Anderson, D.L. (1961). Elastic wave propagation in layered anisotropic media, J. Geophys. Res., 66, 29532963.

Anderson, D.L. (1989). Theory of the Earth, Boston, Blackwell Scientific Publications; http: / / resolver. caltech.edu/CaltechBook:1989.001

Babuska, V., and M. Cara (1991). Seismic anisotropy of the Earth, Boston, Kluwer Acad.

Bhattacharya, S.N. (1976). The extension of ThomsonHaskell method to non-homogeneous spherical layers, Geophys. J. Roy. Astro. Soc., 47, 411-444.

Bhattacharya, S.N., and S. Arora (1997). A flattening transformation for $P-S V$ waves in a transversely isotropic Earth, B. Seismol. Soc. Am., 87, 1297-1304.

Bhattacharya, S.N. (2009). Computation of surface wave velocities in an isotropic non-gravitating spherical Earth using an exact flattening transformation, B. Seismol. Soc. Am., 99, 3525-3528.

Bhattacharya, S.N., S. Mitra and G. Suresh (2013). The shear wave velocity of the upper mantle beneath the Bay of Bengal, Northeast Indian Ocean from interstation phase velocities of surface waves, Geophys. J. Int., 193, 1506-1514.

Bjarnason, I.T., and H. Schmeling (2009). The lithosphere and asthenosphere of the Iceland hotspot from surface waves, Geophys. J. Int., 178, 394-418.

Chen, Y., J. Badal and Z. Zhang (2009). Radial anisotropy in the crust and upper mantle beneath the QinghaiTibet plateau and surrounding regions, J. Asian 
Earth Sci., 36, 289-302.

Dahlen, F.A., and J. Tromp (1998). Theoretical Global Seismology, Princeton, New Jersey, Princeton University Press.

Dziewonski, A.M., and D.L. Andersion (1981). Preliminary reference Earth model, Phys. Earth Planet. In., 25, 297-356.

Ferreira, A.M.G., J.H. Woodhouse, K. Visser and J. Trampert (2010). On the robustness of global radially anisotropic surface wave tomography, J. Geophys. Res., 115, B04313; doi:10:1029/2009JB006718.

Kustowski, B., G. Ekström and A.M. Dziewonski (2008). Anisotropic shear-wave velocity structure of the Earth's mantle: A global model, J. Geophys. Res., 113, B06306; doi:10.1029/2007B005169.

Lebedev, S., J.M.C. Adam and T. Meier (2013). Mapping the Moho with seismic surface waves: A review resolution analysis and recommended inversion strategies, Tectophysics, 609, 377-394.

McEvilly, T.V. (1964). Central US crust-upper mantle structure from Love and Rayleigh wave phase velocity inversion, B. Seismol. Soc. Am., 54, 1997-2015.

Montagner, J.P. (2007). Deep Earth structure - upper mantle structure: Global isotropic and anisotropic elastic tomography, Treatise on Geophysics, vol. 1, Elsevier.

Moschetti, M.P., M.H. Ritzwoller, F. Lin and Y. Yang (2010). Seismic evidence for widespread western-US deep-crustal deformation caused by extension, Nature, 464, 885-889.

Muyzert, E., and R. Snieder (2000). An alternative parameterisation for surface waves in a transverse isotropic medium, Phys. Earth Planet. In., 118, 125133.

Panning, M.P., and B.A. Romanowicz (2006). A threedimensional radially anisotropic model of shear velocity in the whole mantle, Geophys. J. Int., 167, 361-379.

Panning, M.P., V. Lekic and B.A. Romanowicz (2010). Importance of crustal correction in the development of a new global model of radial anisotropy, J. Geophys. Res., 115, B04313; doi:10:1029/ 2010JB007520.

Prajapati, S., G. Suresh and S.N. Bhattacharya (2011). Crustal Structure of the Northwest Deccan Volcanic Province, India and the Adjoining Continental Shelf from Observed Surface Wave Dispersion, B. Seismol. Soc. Am., 101, 1488-1495.

Raykova, R.B., and S.B. Nikolova (2003). Anisotropy in the Earth's crust and uppermost mantle in southeastern Europe obtained from Rayleigh and Love surface waves, J. Appl. Geophys., 54, 247-256.

Shapiro, N.M., and M.H. Ritzwoller (2002). MonteCarlo inversion for a global shear-velocity of the crust and upper mantle, Geophys. J. Int., 151, 88-105. Shapiro, N.M., M.H. Ritzwoller, P. Molnar and V. Levin (2004). Thinning and flow of Tibetan crust constrained by anisotropy, Science, 305, 233-236.

Stein, S., and M. Wysession (2003). An introduction to seismology, earthquakes, and Earth structure, Blackwell Publishing.

Suresh, G., S. Jain and S.N. Bhattacharya (2008). Lithosphere of the Indus block in NW Indian subcontinent through genetic algorithm inversion of surface wave dispersion, B. Seismol. Soc. Am., 98, 1750-1755.

Suresh, G., S.N. Bhattacharya and S.S. Teotia (2015). Crust and upper mantle velocity structure of the northwestern Indian Peninsular Shield from interstation phase velocities of Rayleigh and Love waves, Annals of Geophysics, 58 (2), S0215; doi:10.4401/ag6674.

Takeuchi, H., and M. Saito (1972). Seismic surface waves, In: B.A. Bolt (ed.), Seismology: surface waves and Earth oscillations (Methods in Computational Physics, vol. 11), New York, Academic Press.

Villasenor, A., M.H. Ritzwoller, A.L. Levshin, M.P. Barmin, E.R. Engdahl, W. Spakman and J. Trampert (2001). Shear velocity structure of central Eurasia from inversion of surface wave velocities, Phys. Earth Planet. In., 123, 169-184.

Visser, K, J. Trampert, S. Levedev and B.L.N. Kennett (2008). Probability of radial anisotropy in the deep mantle, Earth Planet. Sci. Lett., 270, 241-250.

Xie, J., M.H. Ritzwoller, W. Shen, Y. Yang, Y. Zheng and L. Zhou (2013). Crustal anisotropy across eastern Tibet and the western Yangtze Craton, J. Geophys. Res., 118, 4226-4252; doi:10.1002/jgrb.50296.

Corresponding author: Sankar N. Bhattacharya, Lithosphere Study Centre, New Delhi, India; email: sn_bhattacharya@hotmail.com.

(C) 2015 by the Istituto Nazionale di Geofisica e Vulcanologia. All rights reserved. 